\title{
THE SCIENTIFIC ORGANIZING COMMITTEE
}

\section{On Behalf of the IAU}

P. Swings, Chairman, Liège University, Belgium

A. Woszczyk, Secretary, Toruń University, Poland

A. Dollfus, Paris Observatory, France

F. D. Drake, Cornell University, Ithaca, U.S.A.

I. Koval, Main Astrophysical Observatory, Kiev, U.S.S.R.

V. I. Moroz, Sternberg Astronomical Institute, Moscow, U.S.S.R.

T. Owen, State University of New York, Stony Brook, U.S.A.

H. C. van de Hulst, Leiden Observatory, The Netherlands

\section{COSPAR Representatives}

A. D. Kuzmin, Academy of Sciences, Moscow, U.S.S.R.

C. Sagan, Cornell University, Ithaca, U.S.A. 